\title{
Bimatoprost ophthalmic solution $0.03 \%$ lowered intraocular pressure of normal-tension glaucoma with minimal adverse events
}

This article was published in the following Dove Press journal:

Clinical Ophthalmology

20 September 2012

Number of times this article has been viewed

\author{
Toyoaki Tsumura' \\ Keiji Yoshikawa ${ }^{2}$ \\ Hirotaka Suzumura ${ }^{3}$ \\ Tairo Kimura ${ }^{4}$ \\ Satoshi Sasaki ${ }^{5}$ \\ Itaru Kimura ${ }^{6}$ \\ Ryuji Takeda ${ }^{7}$
}

'Department of Ophthalmology, Fussa Hospital, Fussa, Tokyo, Japan; ${ }^{2}$ Yoshikawa Eye Clinic, Machida, Tokyo, Japan; ${ }^{3}$ Department of Ophthalmology, Nakano General Hospital, Nakano, Tokyo, Japan; ${ }^{4}$ Ueno Eye Clinic, Ueno, Tokyo, Japan; ${ }^{5}$ Sasaki Eye Clinic, Ueno, Tokyo, Japan; ' $D$ epartment of Ophthalmology, Juntendo University Urayasu Hospital, Urayasu, Chiba, Japan; ${ }^{7}$ Department of Biological Chemistry, Faculty of Agriculture, Kinki University, Nara, Nara, Japan
Correspondence: Toyoaki Tsumura Fussa Hospital, I-6-I Kamidaira,

Fussa, Tokyo I97-85II, Japan

$\mathrm{Tel}+8 \mathrm{I} 4255 \mathrm{I}$ IIII

Fax $+8|4255| 6112$

Email tsumura@fussahsp.jp
Purpose: The aim of this study was to evaluate the efficacy and safety of bimatoprost ophthalmic solution $0.03 \%$ (bimatoprost) in Japanese normal-tension glaucoma (NTG) patients with an intraocular pressure (IOP) of $18 \mathrm{mmHg}$ or less.

Methods: Bimatoprost was instilled into the unilateral conjunctival sac of Japanese NTG patients with a baseline IOP of $18 \mathrm{mmHg}$ or less. The time courses of IOP, conjunctival hyperemia, superficial punctate keratitis, and adverse events were examined at 2, 4, 8, and 12 weeks post bimatoprost instillation.

Results: Thirty-two of the 38 enrolled NTG patients (mean age, $64.1 \pm 12.6$ years; 19 males and 19 females) completed the study, with six patients unable to complete the study (two patients discontinued because of side effects and four patients withdrew). The levels of IOP in the treated eyes were significantly reduced $(P<0.0001)$ from the baseline IOP levels. No significant change in IOP was observed in the fellow eyes. There were significant increases in conjunctival hyperemia. No significant superficial punctate keratitis scores were noted between the baseline and each point examined. Eyelash disorder, eyelid pigmentation, and deepening of the upper eyelid sulcus were observed in 28, six, and three eyes, respectively.

Conclusion: Bimatoprost effectively lowered the IOP. It was well tolerated in Japanese NTG patients, with few patients having to discontinue because of adverse events.

Keywords: intraocular pressure reduction, superficial punctate keratitis, conjunctival hyperemia, antiglaucoma drugs

\section{Introduction}

The lowering of intraocular pressure (IOP) using antiglaucoma drugs is considered the first-line effective therapy for glaucoma treatment. ${ }^{1-4}$ In Japan, as well as in the United States and the European Union, most antiglaucoma drugs have been approved as a result of clinical efficacy against high-IOP glaucoma.

The Tajimi study proved that normal-tension glaucoma (NTG) is the most common type of glaucoma in Japan., 5 The mean IOP in Japanese patients with primary open-angle glaucoma is $14.6 \pm 2.7 \mathrm{mmHg}$; thus the IOP of $92 \%$ of Japanese patients would be $18 \mathrm{mmHg}$ or less, on the premise that the majority of these patients were within the range of the mean plus or minus one standard deviation. Hence, in Japan, the post-marketing evaluation of the usefulness of antiglaucoma drugs, including prostaglandins (PGs), has been required to reduce the IOP of NTG patients; however, few independent clinical studies of antiglaucoma drugs have been conducted. ${ }^{7}$ Bimatoprost ophthalmic solution $0.03 \%$ (bimatoprost) has been marketed in Japan since 2009. Sato et $\mathrm{al}^{8}$ applied latanoprost to NTG patients as a treatment of choice and then 
changed the treatment to bimatoprost; the authors reported the latter had a better effect.

To the best of the present authors' knowledge, this is the first report on the efficacy and safety of bimatoprost being used solely on selected eligible NTG patients.

\section{Material and methods Study design}

This prospective, open-label, multicenter, one-eye study was conducted over a period of 12 weeks at Fussa Hospital, Yoshikawa Eye Clinic, Ueno Eye Clinic, Sasaki Eye Clinic, and Nakano General Hospital, Tokyo, Japan, from April to October in 2010. The study was conducted according to the ethical principles of the Declaration of Helsinki. All subjects read and signed a written informed consent form, and the Institutional Review Board of Fussa Hospital approved the study in advance.

\section{Participants}

All patients were required to read and sign a voluntary, written informed consent form at each hospital and private clinic before participating in this study.

Inclusion criteria were as follows: (1) patients aged 20 years or older who were diagnosed with NTG in both eyes, (2) IOPs of the bilateral eyes were $18 \mathrm{mmHg}$ or less in at least three consecutive measurements (baseline IOP), (3) no history of laser irradiation or surgical treatment of glaucoma; (4) best-corrected visual acuity of 0.5 or less, (5) spherical equivalent refraction of $-10 \mathrm{D}$ or greater, (6) patients who were medicated for glaucoma before study entry were required to undergo a 4-week washout period.

Exclusion criteria were as follows: (1) mean deviation (MD) values using the Humphrey central 30-2/24-2 Swedish Interactive Threshold Algorithm standard program (Carl Zeiss Meditec, Inc, Dublin, CA) was less than $-15 \mathrm{~dB}$, (2) abnormalities of the anterior segment that disturbed accurate IOP measurement using a Goldmann applanation tonometer, (3) apparent findings of dry eye, (4) any active ocular inflammations or blepharitis, (5) patients who use steroid eye drops or those who might use steroid eye drops during the study, (6) ocular fundus diseases that might influence the patient's visual field, (7) patients judged ineligible by each investigator.

\section{Procedure}

After the baseline measurements and observations were made, one drop of bimatoprost was instilled into the conjunctival sac once a day for 12 weeks post bimatoprost instillation (PI), with this done at the same time of day each day for each patient. The contralateral eye with IOP in each subject was followed up without an instillation. The right eye was selected as the treated eye in case the patients had the same IOP bilaterally. Measurements of the IOP, bestcorrected visual acuity, slit-lamp examinations, and adverse event interviews were conducted at the patient's hospital or clinic visits at 2, 4, 8, and 12 weeks PI. The IOP was measured twice between 9 am and $1 \mathrm{pm}$ by a single examiner in each hospital or clinic, using the Goldmann applanation tonometer. The mean of the two IOP measurements at each time point was calculated and used for statistical analysis.

The degree of conjunctival hyperemia was scored from 0 to 3 and the degree of superficial punctate keratitis (SPK) was scored from 0 to 4 , both according to the graded standard photograms based on the cornea diagram of the US National Eye Institute classification system. ${ }^{9,10}$ The authors have used both standard photos for the evaluation in previous studies on other PGs. ${ }^{9,11}$

Each of the adverse events - such as ocular irritation, itching, foreign body sensation, and dry sensation - was classified into four grades: (1) "nothing," (2) "almost nothing," (3) "sometimes," and (4) "always." Side effects such as eyelash growth, eyelid pigmentation, and deepening of upper eyelid sulcus (DUES) were compared using photographs taken before and after treatment with bimatoprost.

Blood pressure and pulse rate were measured at the start and end of the study. MD values were compared before and after the study.

\section{Statistical analysis}

All collected data were sent to Juntendo University Urayasu Hospital, Urayasu, Chiba, Japan, and an ophthalmologist who did not enroll in the study confirmed the diagnosis of NTG based on the presented data, including optic disc photographs and visual field examinations. A statistician at Kinki University, Nara, Nara, Japan, also independently analyzed the data.

IOP levels obtained before the treatment as a baseline and after the treatment with bimatoprost in the subjects who could be followed up for at least 2 weeks were compared. The IOP values obtained from the treated and the fellow eyes were analyzed using a paired $t$-test with Bonferroni correction after repeated analysis of variance. The IOP reduction rate was calculated by dividing the difference in IOP values between the treated and the fellow eyes at each 
time point by the baseline IOP values of the treated eye. The composition ratio of the IOP reduction rate was analyzed by chi-square testing. The IOP-lowering efficacy classified by three different baseline IOP groups (low: $<13 \mathrm{mmHg}$; medium: $13-16 \mathrm{mmHg}$; high: $\geq 16 \mathrm{mmHg}$ ) was also analyzed, using a paired $t$-test with Bonferroni correction after repeated analysis of variance.

Scores of conjunctival hyperemia and SPK were analyzed by a Wilcoxon signed-rank test. Other ocular adverse events were analyzed using a chi-square test. Blood pressures, pulse rates, and $\mathrm{MD}$ values before and after the treatment were compared using the paired $t$-test. JMP software (v 9.0.2; SAS Institute Inc, Cary, NC) was used for statistical analysis. $P$-values less than 0.05 were considered significant.

\section{Results}

Thirty-eight subjects (19 males, 19 females; mean age: $64.1 \pm 12.6$ years) who met the inclusion criteria were included in the study. Table 1 outlines the demographic characteristics of the study participants. All patients were diagnosed as NTG at Juntendo University Urayasu Hospital. Thirty-two of the 38 patients $(84.2 \%)$ completed this study. Six patients discontinued the study: four withdrew and two discontinued because of side effects. One of these patients experienced mild conjunctival hyperemia at 2 weeks PI and wished to discontinue the treatment. Despite the mild cell infiltration in the anterior chamber, another patient resumed instillation of bimatoprost upon her request; this patient's data up to 8 weeks PI were included.

There were no significant differences in the baseline IOP values between the treated and the fellow eyes. The IOP levels in treated eyes were significantly decreased at each time point $(P<0.0001)$. On the contrary, significant lowering was seen only at 8 and 12 weeks PI in the fellow eyes. When the treated eyes were compared with the fellow

Table I Demographic characteristics of the study participants

\begin{tabular}{ll}
\hline Variable & Participants' results \\
\hline Subjects $(\mathrm{n})$ & 38 \\
Sex (male/female) & $19 / 19$ \\
Eyes (R/L) & $38(2 \mathrm{I} / \mathrm{I7})$ \\
Age (years)* & $64.1 \pm \mathrm{I} 2.6$ (range: $33-84)$ \\
RE (D)* & $-1.28 \pm 2.94$ (range: -8.00 to 3.25$)$ \\
MD $(\mathrm{dB})^{*}$ & $-5.22 \pm 4.07$ (range: -14.39 to I.I5) \\
CCT $(\mu \mathrm{m})^{*}$ & $543.4 \pm 38.0$ (range: $474-62 \mathrm{I})$ \\
\hline
\end{tabular}

Note: *Value is presented as the mean plus or minus the standard deviation.

Abbreviations: R, right; L, left; RE, refractive error; $M D$, mean deviation; $\mathrm{CCT}$, central corneal thickness. eyes, IOP levels were significantly decreased at each time point during the study period $(P<0.0001)$ (Figure 1$)$. There were no significant differences in the average reduction rates of IOP in the treated eyes at each time point $(P=0.0859)$. Among them, reduction rates over $20 \%$ were seen in about half of the subjects at each time point (Figure 2). At each time point after the treatment, all three groups classified according to the baseline IOP levels lowered their IOPs significantly (Figure 3 ).

The scores for conjunctival hyperemia in the treated eyes were increased at each time point but they were all less than 1. The score for the fellow eyes remained in the same range (Table 2). No significant differences in the SPK scores were observed at each time point in the treated and the fellow eyes (Table 2). The most common side effects were eyelash growth (87.5\%), eyelid pigmentation (18.8\%), and DUES (9.4\%). The frequently observed adverse ocular symptoms were itching (21.1\%), irritation (15.8\%), foreign body sensation (21.1\%), and dryness (13.2\%). Sixteen of 38 patients $(42.1 \%)$ complained of these adverse events at "sometimes" or "always" during the study period. No apparent systemic adverse reactions such as changes in blood pressure $(104.1 \pm 12.7 \mathrm{mmHg}$ and $105.9 \pm 14.7 \mathrm{mmHg}$, $P=0.3041)$ and pulse rate $(69.5 \pm 9.6$ times per minute and $68.5 \pm 11.1$ times per minute, $P=0.7678$ ) during this treatment were noted. There was no significant difference in $\mathrm{MD}$ values before and after bimatoprost instillation $(-5.22 \pm 4.07 \mathrm{~dB}$ and $-2.72 \pm 4.06 \mathrm{~dB}, P=0.0385)$.

\section{Discussion}

Bimatoprost has a potent IOP-lowering effect in Japanese NTG patients with an IOP of $18 \mathrm{mmHg}$ or less. There were no serious local or systemic adverse events leading to discontinuation of bimatoprost administration.

NTG patients were treated with the intention of lowering IOP levels. Bimatoprost is reported to have a better IOP-lowering effect than latanoprost. ${ }^{12-14}$ Hence, the authors studied the IOP-lowering effect by applying bimatoprost to the unilateral eye and using the contralateral eye as the control in Japanese NTG patients.

The IOP levels of the treated eyes were significantly lower than the baseline IOP values at each time point. Although the IOP levels of the fellow eyes were significantly decreased from the baseline IOP values at 8 and 12 weeks PI, the value of IOP reduction remained at $1.3 \mathrm{mmHg}$. The reduction in IOP observed was significantly greater in the treated eyes than the fellow eyes at all time points, and the efficacy of 


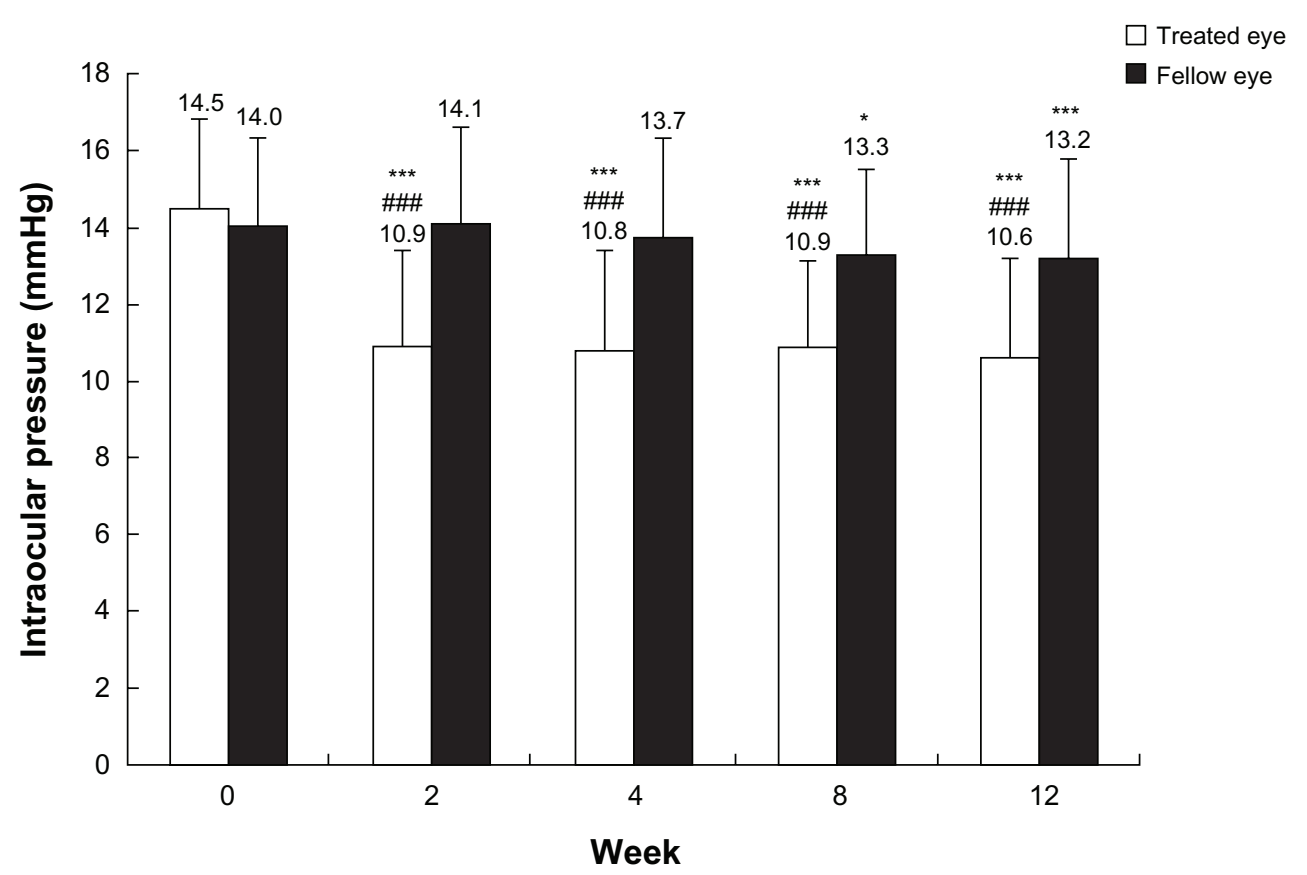

Figure I Intraocular pressure values in treated and fellow eyes.

Notes: Each column represents the mean (plus or minus the standard deviation) intraocular pressure value at each time point; $* P<0.05$; $* * * P<0.00$ I, for each time point versus baseline, as measured by paired $t$-test using Bonferroni correction after repeated analysis of variance; ${ }^{\#}<0.00$ I, for treated eye versus fellow eye at each time point, as measured by unpaired $t$-test using Bonferroni correction after repeated analysis of variance.

IOP lowering in Japanese patients with NTG was evident through this one-eye trial.

A study period of 3 months was chosen because the authors predicted 12 weeks would be required to evaluate the efficacy and safety of a PG analogue. ${ }^{9}$ The mean IOP reduction rates were approximately $20 \%$ from the baseline IOP at all the time points examined, and for $50 \%$ or more patients, IOP lowered $20 \%$ or more from the baseline IOPs. Approximately $20 \%$ of the patients did not reach a $10 \%$ IOP reduction rate from the baseline IOP and these were

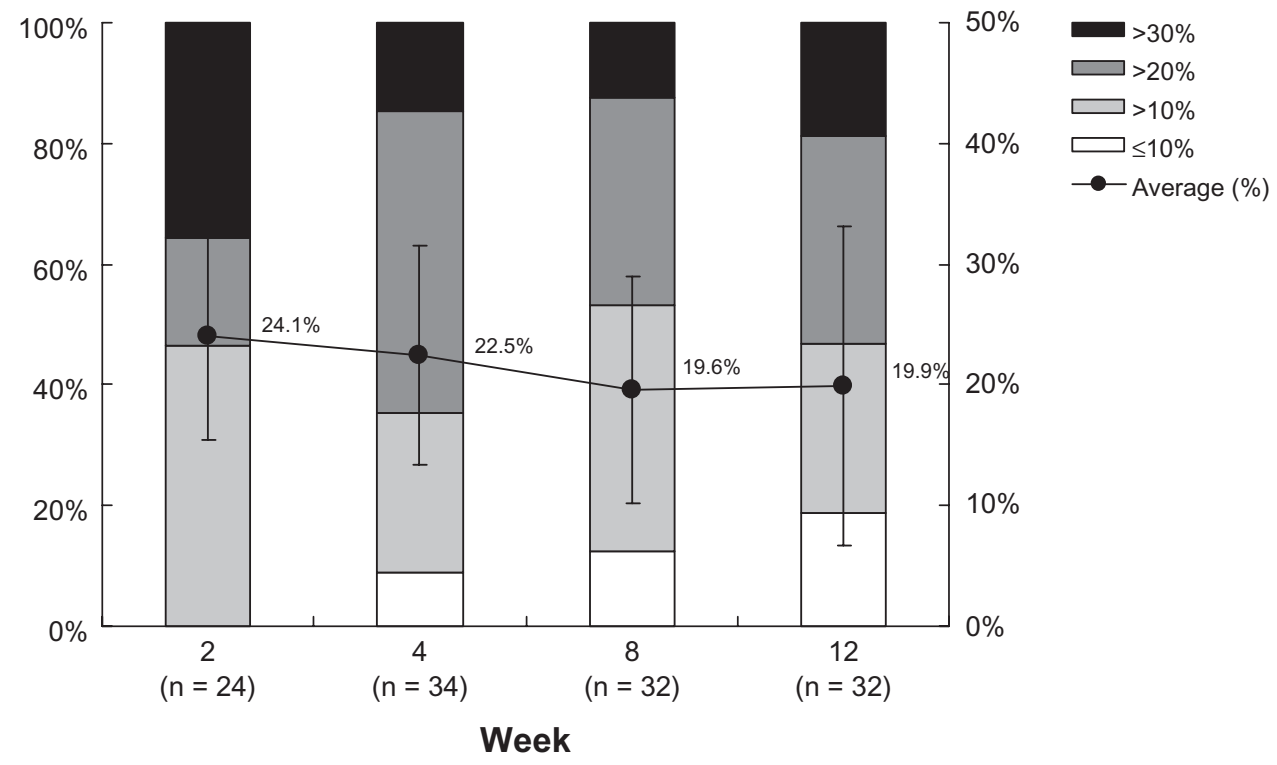

Figure 2 Percentage reduction of intraocular pressure in the treated eyes at each time point.

Notes: Intraocular pressure reduction rates in the treated eyes are classified into four categories: $>30 \%,>20 \%,>10 \%, \leq 10 \%$; closed circle $(\bullet)$ represents the mean plus or minus the standard deviation; no significant difference in distribution at all time points observed, as measured by chi-square test $(P=0.0859)$. 

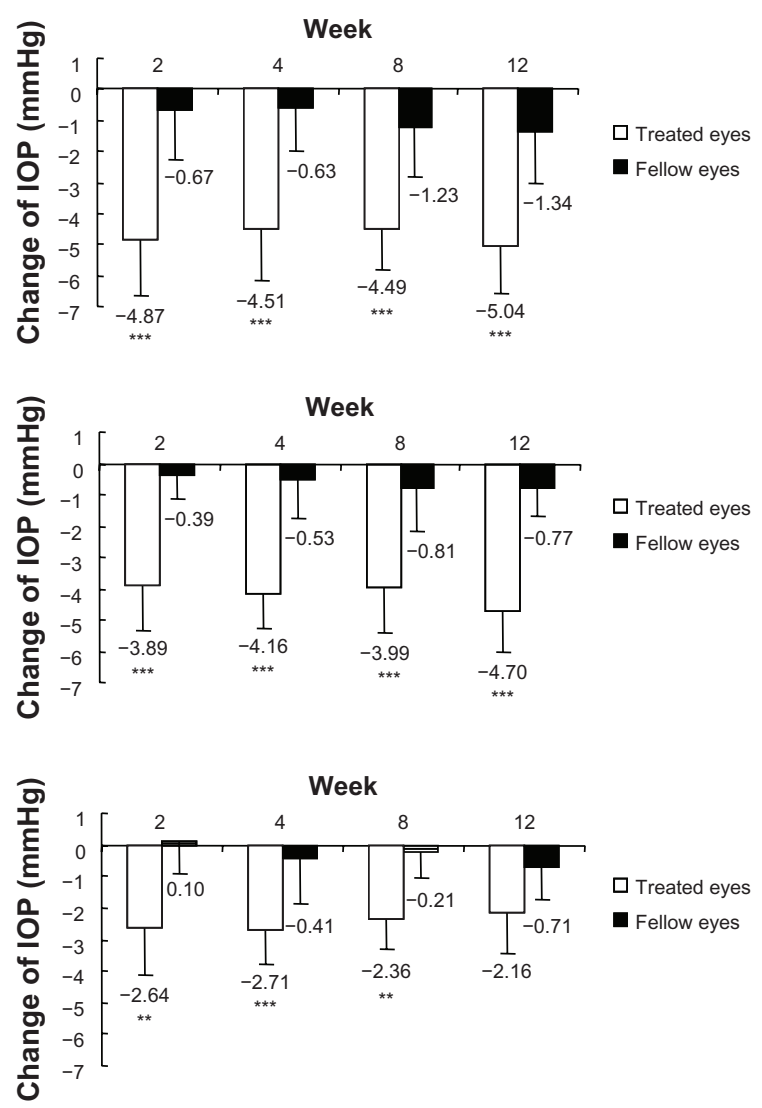

Figure 3 Change in intraocular pressure (IOP) compared with baseline in high, medium, and low groups.

Notes: The treated eyes were classified into the three groups based on their baseline IOP values (low: $<13 \mathrm{mmHg}$; medium: $13-16 \mathrm{mmHg}$; high: $\geq 16 \mathrm{mmHg}$ ); each column represents the mean (plus or minus the standard deviation) of IOP values at each time point; ${ }^{* *} P<0.01$; *** $P<0.00$ I, each time point versus baseline, as measured by unpaired $t$-test using Bonferroni correction after repeated analysis of variance.

considered nonresponders. In general, nonresponders to PGs account for approximately $20 \%$ of glaucoma patients with high IOP. ${ }^{15}$ The rate of "nonresponders" in NTG patients with an IOP of $18 \mathrm{mmHg}$ or less in this study was thought to be similar to that of responders.
When the IOP-lowering efficacy was evaluated by group, with the treated eyes classified into the three groups based on their baseline IOP values (low, medium, and high groups), the IOP levels of the treated eyes in all groups were significantly lowered from the baseline IOP levels. In Japan, about $20 \%$ of NTG eyes have IOP levels equal to $13 \mathrm{mmHg}$ or less. Although the IOP levels of these patients are actually not high, decreasing the IOP levels has been considered a primary therapeutic goal for these patients. Considering these situations, the results given indicate that bimatoprost is an effective treatment of Japanese NTG.

A comparative study of bimatoprost and latanoprost reported that the incidence of conjunctival hyperemia was more than $40 \%$ after bimatoprost instillation and around $20 \%$ after latanoprost instillation. ${ }^{16}$ To evaluate the conjunctival hyperemia, the present authors viewed the standard photograms of the subjects during the study period. The incidence and severity of conjunctival hyperemia with bimatoprost was lower than in the previous studies. ${ }^{16,17}$ Conjunctival hyperemia may discourage patients from continuous treatment with bimatoprost. ${ }^{17}$ It is important for patient adherence to the treatment that the possible hyperemic side effects are explained to patients in advance.

SPK caused by latanoprost ophthalmic solution containing benzalkonium chloride has been reported. ${ }^{11}$ The low incidence of SPK in those receiving the bimatoprost treatment may be because of lower concentration of benzalkonium chloride in the ophthalmic solution. ${ }^{18}$

Under continuous usage of PGs, adverse reactions such as eyelash growth, eyelid pigmentation, and DUES are thought to be inevitable. ${ }^{19}$ Eyelid washing immediately after instillation is necessary during PG treatment.

In this study, adverse events were observed in 16 of the 38 subjects. However, the authors considered that the frequency of these adverse events was not high and that the adverse events remained mild and clinically tolerable.

Table 2 Time course of conjunctival hyperemia and superficial punctate keratitis (SPK) scores

\begin{tabular}{|c|c|c|c|c|c|}
\hline \multirow[t]{2}{*}{ Score } & \multicolumn{5}{|c|}{ Study period } \\
\hline & Baseline & 2 weeks & 4 weeks & 8 weeks & 12 weeks \\
\hline \multicolumn{6}{|c|}{ Conjunctival hyperemia } \\
\hline Treated eyes & $0.26 \pm 0.45$ & $0.7 I \pm 0.60$ & $0.79 \pm 0.54$ & $0.88 \pm 0.55$ & $0.94 \pm 0.44$ \\
\hline Fellow eyes & $0.24 \pm 0.43$ & $0.11 \pm 0.31$ & $0.18 \pm 0.46$ & $0.22 \pm 0.49$ & $0.25 \pm 0.44$ \\
\hline$P$-value & 0.5706 & $<0.0001$ & $<0.0001$ & $<0.0001$ & $<0.0001$ \\
\hline \multicolumn{6}{|l|}{ SPK } \\
\hline Treated eyes & $0.07 \pm 0.17$ & $0.09 \pm 0.19$ & $0.06 \pm 0.13$ & $0.05 \pm 0.11$ & $0.11 \pm 0.18$ \\
\hline Fellow eyes & $0.07 \pm 0.19$ & $0.04 \pm 0.16$ & $0.02 \pm 0.06$ & $0.03 \pm 0.09$ & $0.06 \pm 0.13$ \\
\hline$P$-value & 1.0000 & 0.2919 & 0.0894 & 0.4664 & 0.1992 \\
\hline
\end{tabular}

Notes: Scores are presented as the mean plus or minus the standard deviation; analyzed by Wilcoxon signed-rank test. 


\section{Conclusion}

The results of this study show that bimatoprost lowered the IOP in the eyes of Japanese NTG patients at the rate of around $20 \%$ from the baseline IOP, with a small number of side effects and adverse events including conjunctival hyperemia observed. These results indicate that bimatoprost is a potent tool in the treatment of NTG in patients with an IOP of $18 \mathrm{mmHg}$ or less.

\section{Disclosure}

This study was performed without support from any associations or companies. The authors report no conflicts of interest in this work.

\section{References}

1. Collaborative Normal-Tension Glaucoma Study Group. The effectiveness of intraocular pressure reduction in the treatment of normal-tension glaucoma. Am J Ophthalmol. 1998;126(4):498-505.

2. Leske MC, Heijl A, Hussein M, Bengtsson B, Hyman L, Komaroff E; for Early Manifest Glaucoma Trial Group. Factors for glaucoma progression and the effect of treatment: the Early Manifest Glaucoma Trial. Arch Ophthalmol. 2003;121(1):48-56.

3. Advanced Glaucoma Intervention Study Investigators. The Advanced Glaucoma Intervention Study (AGIS): 7. The relationship between control of intraocular pressure and visual field deterioration. Am J Ophthalmol. 2000;130(4):429-440.

4. Kass MA, Heuer DK, Higginbotham EJ, et al. The Ocular Hypertension Treatment Study: a randomized trial determines that topical ocular hypotensive medication delays or prevents the onset of primary openangle glaucoma. Arch Ophthalmol. 2002;120(6):701-713.

5. Iwase A, Suzuki Y, Araie M, et al; for Tajimi Study Group, Japan Glaucoma Society. The prevalence of primary open-angle glaucoma in Japanese: the Tajimi Study. Ophthalmology. 2004;111(9):1641-1648.

6. Yamamoto T, Iwase A, Araie M, et al; for Tajimi Study Group, Japan Glaucoma Society. The Tajimi Study report 2: prevalence of primary angle closure and secondary glaucoma in a Japanese population. Ophthalmology. 2005;112(10):1661-1669.

7. Araie M, Kitazawa Y. Long-term efficacy and safety of $0.03 \%$ bimatoprost ophthalmic solution in patients with primary open-angle glaucoma or ocular hypertension. Atarashii Ganka. 2011;28(8):1209-1215. Japanese.
8. Sato S, Hirooka K, Baba T, et al. Efficacy and safety of switching from topical latanoprost to bimatoprost in patients with normal-tension glaucoma. J Ocul Pharmacol Ther. 2011;27(5):499-502.

9. Nakano T, Yoshikawa K, Kimura T, Suzumura H, Nanno M, Noro T. Efficacy and safety of tafluprost in normal-tension glaucoma with intraocular pressure of $16 \mathrm{mmHg}$ or less. Jpn J Ophthalmol. 2011;55(6):605-613.

10. Lemp MA. Report of the National Eye Institute/Industry workshop on clinical trials in dry eyes. CLAO J. 1995;21(4):221-232.

11. Yamazaki S, Nanno M, Kimura T, Suzumura H, Yoshikawa K. Effects of switching to SofZia-preserved travoprost in patients who presented with superficial punctate keratopathy while under treatment with latanoprost. Jpn J Ophthalmol. 2010;54(1):7-14.

12. Cheng JW, Wei RL. Meta-analysis of 13 randomized controlled trials comparing bimatoprost with latanoprost in patients with elevated intraocular pressure. Clin Ther. 2008;30(4):622-632.

13. Fiscella R, Walt J. Estimated comparative costs of achieving a $20 \%$ reduction in intraocular pressure with bimatoprost or latanoprost in patients with glaucoma or ocular hypertension. Drugs Aging. 2006;23(1): $39-47$.

14. Gandolfi S, Simmons ST, Sturm R, Chen K, VanDenburgh AM; for Bimatoprost Study Group 3. Three-month comparison of bimatoprost and latanoprost in patients with glaucoma and ocular hypertension. $A d v$ Ther. 2001;18(3):110-121.

15. Ikeda Y, Mori K, Ishibashi T, Naruse S, Nakajima N, Kinoshita S. Latanoprost nonresponders with open-angle glaucoma in the Japanese population. Jpn J Ophthalmol. 2006;50(2):153-157.

16. Kurtz S, Mann O. Incidence of hyperemia associated with bimatoprost treatment in naïve subjects and in subjects previously treated with latanoprost. Eur J Ophthalmol. 2009;19(3):400-403.

17. Honrubia F, García-Sánchez J, Polo V, de la Casa JM, Soto J. Conjunctival hyperaemia with the use of latanoprost versus other prostaglandin analogues in patients with ocular hypertension or glaucoma: a meta-analysis of randomised clinical trials. $\mathrm{Br}$ J Ophthalmol. 2009;93(3):316-321.

18. Noecker RJ, Herrygers LA, Anwaruddin R. Corneal and conjunctival changes caused by commonly used glaucoma medications. Cornea. 2004;23(5):490-496.

19. Filippopoulos T, Paula JS, Torun N, Hatton MP, Pasquale LR, Grosskreutz CL. Periorbital changes associated with topical bimatoprost. Ophthal Plast Reconstr Surg. 2008;24(4):302-307.
Clinical Ophthalmology

\section{Publish your work in this journal}

Clinical Ophthalmology is an international, peer-reviewed journal covering all subspecialties within ophthalmology. Key topics include: Optometry; Visual science; Pharmacology and drug therapy in eye diseases; Basic Sciences; Primary and Secondary eye care; Patient Safety and Quality of Care Improvements. This journal is indexed on Submit your manuscript here: http://www.dovepress.com/clinical-ophthalmology-journal

\section{Dovepress}

PubMed Central and CAS, and is the official journal of The Society of Clinical Ophthalmology (SCO). The manuscript management system is completely online and includes a very quick and fair peer-review system, which is all easy to use. Visit http://www.dovepress.com/ testimonials.php to read real quotes from published authors. 